\title{
Use of Platelet rich plasma in chronic wounds by a quantification protocol
}

\begin{abstract}
Introduction: Platelet-rich plasma is an autologous concentration of human platelets in a small volume of plasma that represents an increase in platelets compared to normal basal concentrations, making it a source of easy access to the growth factors contained in them. It has a $\mathrm{pH}$ between 6.5 and 6.7. It comes from the patient's own blood, so it is free of contagious diseases and cannot cause hypersensitivity reactions. The platelet count of optimal platelet-rich plasma is debatable. According to the Spanish Agency for Medicines and Health Products, (AEMPS), it must contain a platelet count higher than the baseline serum concentrations considered normal; between 200,000 and 450,000 platelets / $\mathrm{mm} 3$, but another authors, consider a platelet rich plasma of quality when the amount of platelets obtained in the final product exceeds $1,000,000 / \mathrm{mm} \cdot .^{3-5}$ The aim of the present study was to implement a standardized protocol for the quantification of platelet-rich plasma for use in chronic wounds.
\end{abstract}

Methods: Forty patients were included for the study, divided into two groups, both sexes $19(48 \%)$ male and 21 (52\%) female, between 40 and 70 years of age, with an average of 51 years, median of 51 to 54 years and fashion of 54 years. There were 18 patients treated by wound dehiscence, 10 with venous ulcer, 8 with diabetic foot ulcer, 3 with soft tissue infection and 1 with traumatic wound.

Results: According to the results obtained, there was a significantly greater difference in recovery time ( $20 \%$ less than control group) and healing $(46 \%)$ the clinical characteristics appreciated in control group with a SD 1.28 in depth, 4.4 in long and 5.5 in width compared with experimental group with a SD .245 in depth, 2.950 in long, and 3.3 in width. This led to a shorter in-hospital stay and a decrease in the number of subsequent surgical treatments. This led to a shorter in-hospital stay and a decrease in the number of subsequent surgical treatments.

Conclusions: The patients reported less pain and improvement observed in a short time obtaining reincorporation to their daily activities, shortening in the time of evolution, and lengthening the application of therapies. The applications of the PRP are diverse, being able to apply to almost any type of wound generated because of accidents or surgical interventions, in burned patients, with dermal lesions and / or in the operative and postoperative process.

Keywords: platelet rich-plasma, chronic wounds, quantification protocol, otolaryngology, plastic surgery, dermatology, general surgery, ophthalmology, obstetrics, gynecology, neurosurgery, mitochondria, peroxisomes, ribosomes
Volume 2 Issue 5 - 2018

Carrillo Rivera Jorge Arnulfo,' Lorena Medrano Avilés, ${ }^{2}$ Sonia Guadalupe Hernández Bocanegra, ${ }^{2}$ Tolentino González Christian Stefan, ${ }^{3}$ Flores Luis Rodrigo, ${ }^{4}$ González Masso José Gerardo, ${ }^{4}$ Aguirre Solorio Berenice, ${ }^{4}$ Inzunza Estrada Héctor, ${ }^{4}$ Hernández Ruiz Elliot Jonathan, ${ }^{5}$ Caltenco Solís Raúl Beder, ${ }^{5}$ González López Annel Ivonne, ${ }^{5}$ Buendía García Ana Laura, ${ }^{5}$ Radilla Flores Mariana del Carmen, ${ }^{5}$ Daniel Flores Rodríguez, ${ }^{6}$ Mario Hernandez Rivera ${ }^{7}$ 'Oral and Maxillofacial Surgery, General Hospital, Professor in Justo Sierra University, Mexico

${ }^{2}$ Nurse with master degree in Diagnosis and Management of Chronic Wounds, Mexico

${ }^{3}$ General Surgeon, General Hospital Darío Fernández Fierro, ISSSTE, Mexico

${ }^{4}$ Oral and Maxillofacial Surgeon, Private practice, Mexico ${ }^{5}$ Resident in General Surgery, General Hospital Darío Fernández Fierro, ISSSTE, Mexico

${ }^{6}$ Resident in Plastic and Reconstructive Surgery, CMN, 20 de Noviembre ISSSTE, Mexico

${ }^{7}$ Biomedical engineer, Mexico

Correspondence: Carrillo Rivera Jorge Arnulfo, Oral and Maxillofacial Surgery, General Hospital “Dr. Darío Fernandez Fierro" ISSSTE, Professor in Justo Sierra University, Private practice Mexico City, Mexico, Email dr_jorge_carrilla_cmf@ hotmail.com

Received: September II, 2018 | Published: October 17, 2018

\section{Introduction}

The use of platelet-rich plasma has become a technique increasingly used in various areas of medicine. From its origins of use in stomatological medicine and dental implantology in the mid-1980s, it has progressively expanded its field of use in clinical specialties as diverse as Otolaryngology, Plastic Surgery, Dermatology, General Surgery, Ophthalmology, Obstetrics, Gynecology, and Neurosurgery, among other. Platelets are anucleated cell fragments derived from the cytoplasm of the megakaryocytes of the bone marrow. Traditionally its best known function is in the process of primary hemostasis, because they are indispensable for the formation of the clot; however, they also play an important role in inflammation, immunity, tumor progression and, of course, thrombosis. Platelets contain diverse organelles: mitochondria, peroxisomes, ribosomes, as well as glycogen and granules; the latter are divided into three types: 1) alpha: containing fibrinogen, von Willebrand factor, platelet-derived growth factor, ectodermal growth factor, vascular endothelial growth factor, insulin growth factor type 1, as well as other growth factors. 2) delta: containing ADP, ATP, serotonin, adrenaline, noradrenaline and dopamine and 3) lambda: which are lysosomes that help dissolve the clot once it has fulfilled its function. ${ }^{1-3}$ In addition, to the functions of platelets, recent discoveries regarding their capacity for protein synthesis, containing mRNA copies of almost a third of the known proteins in the human genome, despite lacking a nucleus, some nongenomic functions of these factors are also investigated, such as their effect on signaling pathways, that involve platelet activation, and their role in de novo synthesis, of pro and anti-inflammatory factors.

The enormous amount of growth factors contained in the platelet alpha granules, the capacity for de novo synthesis of proteins, as well as its microbicidal and inflammatory modulating activity favor cell proliferation and immunomodulation and the synthesis of extracellular matrix, promoting healing, the repair of wounds and other tissue injuries. These functions have led us to propose the use of platelet-rich plasma for the repair and regeneration of various tissues. 
The method of obtaining and preparing platelet-rich plasma varies depending on the author, a single procedure or double centrifugation, the time, as well as the type of filter used, of which currently available of more than 40 in the market. Regarding temperature, according to the majority of experts consulted, for the production of a platelet-rich plasma, the optimum preparation temperature during the procedure should be 16 to $22{ }^{\circ} \mathrm{C}$. This temperature range is the one with the highest capacity for platelet concentration and growth factors, because it maintains greater survival of the platelet, regardless of the type of procedure and filter, used with a mean platelet count of 1,150,000 / $\mathrm{mm}^{3}$ (range: $750,000-1,500,000 / \mathrm{mm} 3$ ), as well as, concentrations of platelet and plasma growth factors, between five and seven times higher than the normal concentrations found in peripheral blood. ${ }^{6}$

Depending on the type of filter or pipetting and centrifugation procedure used, different plasma components can be obtained; for example: plasma rich in platelets and plasma growth factors, plasma rich in platelets and poor in plasma growth factors, plasma rich in growth factors and poor in platelets or platelet-rich plasma and leukocytes. There is not a clear correlation between the capacity of greater platelet concentration and the concentrations of platelet growth factors determined in the final product, regardless of the type of filter and the procedure used to prepare it. No difference was observed in the final product obtained regardless of the type of procedure and filter used in relation to the age and sex of the patient either. According to the most recent studies, leucocyte-rich plasmas contain higher concentrations of growth factors such as VEGF and TGF-B, while in platelet-rich plasma without leukocyte layer use it would be possible to concentrate a greater number of growth factors of platelet origin and IGF-1. ${ }^{6}$ Regarding the cellular part contained in the plateletrich plasma, in the platelet-rich plasma rich in leukocytes with use of the Buffy-Coat fraction of the final centrifugation, the leukocyte concentration increases three and five times more than in peripheral blood with predominance of mononuclear cells $(90 \%$ of the total leukocyte, up to $15 \%$ of them with positive labeling for CD 34). Of all the methods of obtaining, four procedures stand out from the others, because they are the most standardized and used by the majority of authors. Two of them use a double centrifugation system, while in the other two the centrifugation procedure is unique Table $1 .{ }^{7,8}$

Table I Methods of centrifugation system

\begin{tabular}{|c|c|c|c|c|}
\hline $\begin{array}{l}\text { Procedure } \\
\text { centrifugation }\end{array}$ & Author & Centrifugation & Double & $\begin{array}{l}\text { Average platelet-rich } \\
\text { plasma quantification }\end{array}$ \\
\hline I & García et al. $^{6}$ & I,800 rpm, 8 minutes & I,800 rpm, 8 minutes & $191 \%$ \\
\hline 2 & Anitua and Andía & I,800 rpm, 8 minutes & No & $90 \%$ \\
\hline \multirow[t]{2}{*}{3} & Okuda and cols & $2,400 \mathrm{rpm}, 10$ minutes & $3,600 \mathrm{rpm}, \mathrm{I} 5$ minutes & $32 \%$ \\
\hline & Kawase and cols & & & \\
\hline 4 & $\begin{array}{l}\text { Deobarrio and cols } \\
\text { Camargo and cols }\end{array}$ & $5,600 \mathrm{rpm}, 6$ minutes & No & $5 \%$ \\
\hline
\end{tabular}

\section{Standardized method}

In the initial appointment, the data collection was performed on a text sheet with the characteristics of the wound, diameter and depth, as well as the general data of the patient, systemic diseases, allergic background, prior informed consent, a photographic sample of the patient was taken. Washing the wound with injectable water and liquid and neutral surgical soap with a $\mathrm{pH}$ of 7 (benzalkonium chloride), removing soapy excess with injectable water, eliminating devitalized tissue, drying the wound bed with sterile gauze was performed obtaining blood from the patient with aseptic technique, from the radial or ulnar vein to process in the centrifuge and obtain the plasma divided into 4 states: supernatant, liquid, clot, autologous fibrin and in two fractions, a platelet-rich fraction and a platelet-poor fraction. The method of obtaining and preparing platelet-rich plasma was routinely carried out with the use of the BD vacutainer ${ }^{\mathrm{TM}}$ system consisting of a sterile double-ended needle with a retractable sleeve that allows multiple takings, a plastic tube with predetermined vacuum with $3.2 \%$ sodium citrate as an anticoagulant that allows obtaining the necessary volume of blood maintaining the adequate additive-sample ratio, avoiding the presence of preanalytical errors in the sample and a holder or support that allows the system to be maintained completely. The amount of autologous blood obtained varies depending on the size and depth of the wound Figures $1 \& 2$. Performing a single centrifugation procedure, with a time of 3 minutes at 1800 RPM according to the calibration and quantification of platelets in the Horiba ABX Micros ES60 тм hematological analyzer performing a precise cell identification using the electronic impedance variation method printing the curves of erythrocyte counts (RBC), leukocytes (WBC) and platelets (PLT) Figures $3 \& 4$.
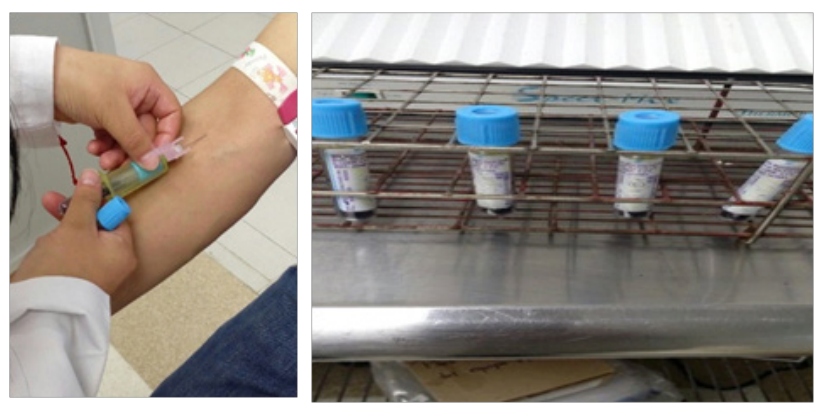

Figures I \& 2 Method of obtaining autologous bleeding and placement in tubes with trisodium citrate as an anticoagulant.

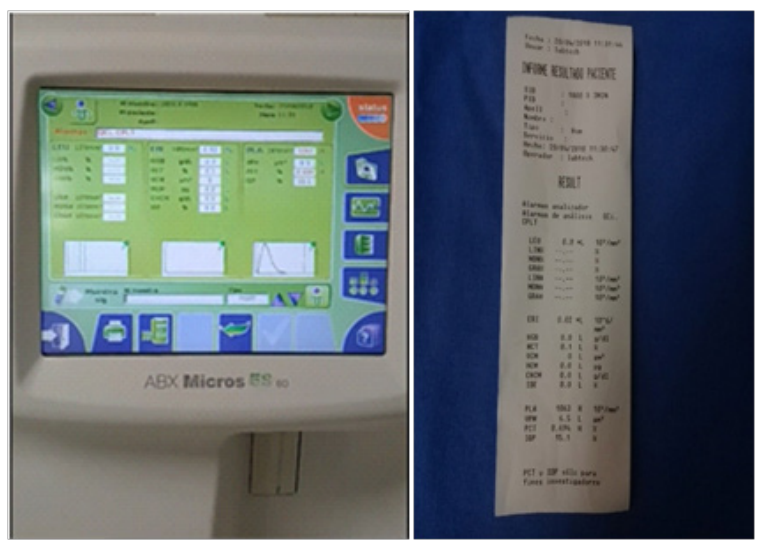

Figures 3 \& 4 Hematological analyzer and impression of platelet counts per cubic millimeter. 
This time interval of 3 minutes at 1800 revolutions was the one with the highest capacity for platelet concentration, maintaining a greater survival of the platelet, with an average platelet count of 800,000 / mm3 (range: 750,000-950,000 / mm3). Activation of platelet-rich plasma was achieved by adding $10 \%$ calcium gluconate with a direct pipetting technique at a ratio of 1 to 1, i.e. 10 drops of gluconate calcium per 1 cubic centimeter of plasma Figures 5 $\&$ 6. After activating the platelet-rich plasma presented in a liquid therapeutic formulation, maintaining a constant temperature of approximately $37.5{ }^{\circ} \mathrm{C}$ for a time of between 3 to 10 minutes was achieved the formation of a clot formed by a three-dimensional fibrin matrix and components cell phones soaked in growth factors. The third phase consists of subsequent appointments with the PRP application allowing to measure again the depth and the length of the wound with respect to the previous appointment, during the subsequent control the application of the platelet-rich plasma can be performed again to allow the wound has re epithelialization and de novo synthesis of pro and anti-inflammatory growth factors Figures $7 \& 8$.
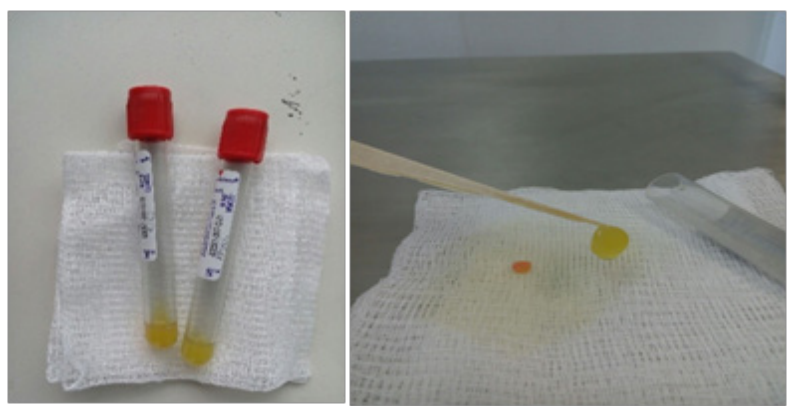

Figures 5 \& 6 Activation of platelet-rich plasma with calcium gluconate pipetting technique with direct.

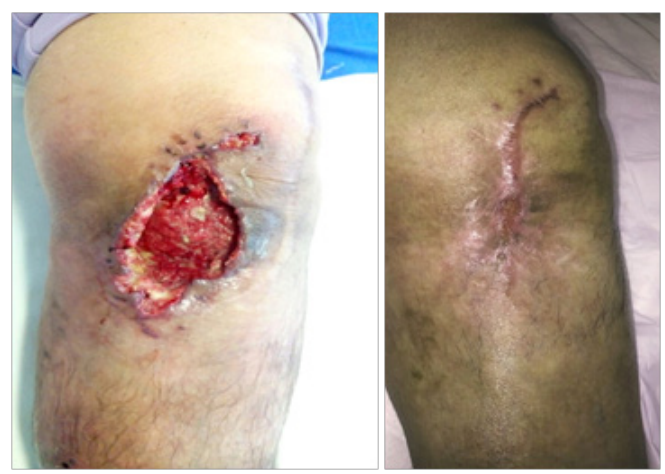

Figure 7 \& 8 Clinical photographs of avulsion wound in the left knee before and after the application of platelet-rich plasma at three weeks of evolution.

\section{Materials and methods}

Healthy patients or with controlled systemic diseases of both sexes aged between 40 and 70 years with chronic non-infected wounds, who are not under anticoagulant treatment, without autoimmune diseases, blood dyscrasias or benign or malignant tumors were included for the study. Forty seven patients divided in two groups, in the control group, 20 patients of both sexes 10 male (50\%) and 10 female (50\%), in the experimental group, 27 patients of which were 14 male patients $(51.8 \%)$ and 13 female patients (48.1\%) among 40 and 70 years old, with an average of 51 years, median of 51 to 54 years and fashion of 54 years.

\section{Results}

Forty patients were included for the study, divided into two groups, both sexes 19 (48\%) male and 21 (52\%) female, between 40 and 70 years of age, with an average of 51 years, median of 51 to 54 years and fashion of 54 years. There were 18 patients treated by wound dehiscence, 10 with venous ulcer, 8 with diabetic foot ulcer, 3 with soft tissue infection and 1 with traumatic wound Graph 1 . According to the results obtained, 19 patients included in the experimental group with the use of platelet-rich plasma, there was a significantly greater difference in recovery time (20\% less than control group) and healing (46\%) according to the clinical characteristics appreciated in control group with a SD 1.28 in depth, 4.4 in long and 5.5 in width compared with experimental group with a SD .245 in depth, 2.950 in long, and 3.3 in width. This led to a shorter in-hospital stay and a decrease in the number of subsequent surgical treatments (Table 2) and (Table 3).

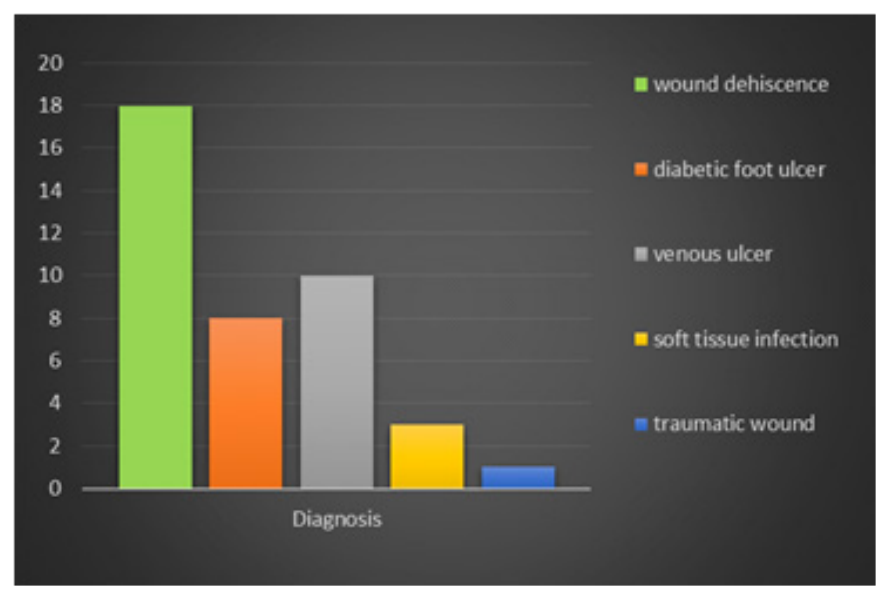

Graph I Both groups divided by sex and age and diagnostic.

Table 2 Descriptive statistics control group

\begin{tabular}{llllll}
\hline & Number of patients & Minimum measure & Maximum measure & Media & Standard deviation (P) \\
\hline Depth & 20 & $1 \mathrm{~cm}$ & $4 \mathrm{~cm}$ & 1.28 & 1.019 \\
Long & 20 & $1 \mathrm{~cm}$ & $16 \mathrm{~cm}$ & 7.11 & 4.459 \\
Width & 20 & $2 \mathrm{~cm}$ & $20 \mathrm{~cm}$ & 7.68 & 5.559 \\
Valid number (By List) & 20 & & & &
\end{tabular}


Table 3 Descriptive statistics experimental group

\begin{tabular}{llllll}
\hline & Number of patients & Minimum measure & Maximum measure & Media & Standard deviation (P) \\
\hline Depth & 19 & 0 & $1 \mathrm{~cm}$ & 0.57 & 0.245 \\
Long & 19 & $1 \mathrm{~cm}$ & $12 \mathrm{~cm}$ & 3.74 & 2.95 \\
Width & 19 & $1 \mathrm{~cm}$ & $13 \mathrm{~cm}$ & 3.16 & 3.367 \\
Valid number (By List) & 19 & & & & \\
\hline
\end{tabular}

\section{Discussion}

The applications of the PRP are diverse, being able to apply to almost any type of wound generated as a result of accidents or surgical interventions, however, patients with an increased risk of infection and in burned patients, with skin lesions and / or in the operative process and postoperative. Because platelet-rich plasma promotes accelerated tissue regeneration, the results obtained help reduce recovery time by $20 \%$ in 20 patients. Platelet-rich plasma is an effective therapy given that it is rich in growth factors, offers modulating properties, stimulates the proliferation of cells and decreases the degree of inflammation, thus allowing wound closure by second intention to be much faster and effective $(46 \%)$. This treatment, which initially used in the fields of maxillofacial surgery due to the stimulating effects on fibroblasts of the periodontal structure, has acquired special relevance in the care and treatment of wounds difficult to heal for the benefit of tissue regeneration. At the Darío Fernández Hospital Wound Clinic, this treatment performed on 39 patients with different diagnoses and clinical characteristics. Given the complexity as well as the different planimetry and the depth of the wound, for 4 to 20 weeks the therapy applied. The patients reported less pain and improvement observed in a short time obtaining reincorporation to their daily activities, shortening in the time of evolution, and lengthening the application of therapies.

\section{Conclusion}

Treatment with platelet-rich plasma is a viable alternative for the recovery of the patient suffering from a chronic wound or difficult to heal. Given the complexity as well as the different planimetry and the depth of the wound, the therapy was applied for 4 to 20 weeks. The patients reported less pain and improvement was observed in a short time obtaining reincorporation to their daily activities, shortening in the time of evolution, and lengthening the application of therapies. The applications of the PRP are diverse, being able to apply to almost any type of wound generated as a result of accidents or surgical interventions, in burned patients, with dermal lesions and / or in the operative and postoperative process.

\section{Ethical aspects}

This study was carried out in accordance with the Declaration of Helsinki and the provisions of the General Health Law regarding research on Human Beings in Mexico. According to the General Health Law, considering Art. 3, 14 and 17 that includes it within the category: Research with minimal risk, because they will use common procedures such as anthropometric measurements, biochemical measurements and diet treatment. -Routine therapeutic. Likewise, based on Art 22 of the same Law, informed consent will be requested in writing to the patient.

\section{Acknowledgements}

None.

\section{Conflict of interest}

The author declares there in so conflict of interest.

\section{References}

1. González Lagunas J. Plasma rico en plaquetas. Revista Española de Cirugía Oral y Maxilofacial. 2006;28(2):89-99.

2. Fernández-Fairen M. Evidencia científica de los factores de crecimiento en cirugía ortopédica y traumatología. Revista Orthotips. 2004;10(4):235-257.

3. González, Maczy, Arteaga-Vizcaíno, et al. Aplicación del plasma rico en plaquetas (PRP) y sus derivados en implantología dental y cirugía plástica. Investigación Clínica. 2012;53(4):408-418.

4. Norma Oficial Mexicana Para La Transfusión Sanguínea. 2006.

5. Rodríguez Flores, Jordi, Palomar Gallego, et al. Plasma rico en plaquetas: fundamentos biológicos y aplicaciones en cirugía maxilofacial y estética facial. Revista Española de Cirugía Oral y Maxilofacial. 2012;34(1):817.

6. Acosta AP, Gutiérrez SJ, Bedoya MA, García DA, Moreno XE. Evaluación del efecto del plasma rico en plaquetas en diferentes tiempos y concentraciones sobre la proliferación de fibroblastos de ligamento periodontal y osteoblastos. Univ Odontol. 2017;36(76).

7. Reyes MM. Actualización de la técnica de Obtención y uso del Plasma Rico en Factores de Crecimiento (P.R.G.F.). Revista Dental de Chile. 2002;93:25-28.

8. Moreno, Raquel, Gaspar Carreño, et al. Técnicas de obtención del plasma rico en plaquetas y su empleo en terapéutica osteoinductora. Farmacia Hospitalaria. 2015;39(3):130-136. 